\title{
Metal ion uptake behavior of nafion in presence of organic complexing reagents
}

\begin{abstract}
In the present study, uptake studies of transition metal ions like $\mathrm{Cu}^{2+}$ and $\mathrm{Co}^{2+}$ were carried out with Nafion membrane. The effects of different experimental conditions were evaluated. The Nafion membrane was pre-treated by two different ways and used for the studies. The studies were also carried out in presence of complexing agents like ethylenediamine and EDTA. It was seen that the maximum uptake occurred in the $\mathrm{pH}$ range of 3-5. The uptake capacity of Nafion was affected by the pre-treatment procedure. This was attributed to the changes of Nafion structure. The presence of different Complexing agents in solution affects the uptake capacity. This could be understood from the strength of the complexes formed by metal ions and thus the reduction in the uptake capacity. The formation of negatively charged complexes by EDTA results in a very low uptake of the metal ions.
\end{abstract}

Keywords: nafion, transition metal ions, uptake rate, complexing agents
Volume I Issue 7 - 2017 Jayshree Ramkumar, Chandramouleeswarana
S
Analytical Chemistry Division, Bhabha Atomic Research Centre, India

Correspondence: Jayshree Ramkumar, Scientific officer, Analytical Chemistry Division, Bhabha Atomic Reasearch Centre, Trombay Mumbai- 400085 India, Tel +9I22 2559 2224, Email jrk@barc.gov.in

Received: November 24, 2017 | Published: December 19, 2017

\section{Introduction}

Membrane separation is a very well known technology having a large number of applications. Separation of metal ions by selective membrane transport is of great importance from both fundamental and practical viewpoints. According to IUPAC, a membrane is defined as a heterogeneous phase which acts as a barrier to the flow of molecules and ionic species in liquid or vapour phase. Membranes can be classified into solid and liquid membranes. Ionomers are a class of solid ion exchange membranes with ionic content as high as 10$15 \mathrm{~mol} \%$. The most common ionomer is Nafion 117. It is a sulfonated tetrafluoroethylene based fluoropolymer-copolymer possessing excellent water sorption and metal ion transport properties. ${ }^{1}$ Cellulose nitrate membrane was also used for sorption of metal ions but the solubility of the membrane in acid was used as an advantage in preconcentration techniques for the determination metal ions. A membrane filtration procedure applied for the preconcentration of nickel(II), cadmium (II), copper(II), cobalt(II) and lead(II) ions in which the metal ions were adsorbed on cellulose nitrate membrane filter as their ammonium pyrrolydinedithiocarbamate (APDC) complexes. Then membrane filter was dissolved by using nitric acid for quantification. ${ }^{2}$ The metal-calmagite complexes on a soluble cellulose nitrate membrane filter were tried for the preconcentration of $\mathrm{Cr}(\mathrm{III}), \mathrm{Co}(\mathrm{II}), \mathrm{Cu}(\mathrm{II}), \mathrm{Fe}(\mathrm{III})$ and $\mathrm{Pb}(\mathrm{II})$ ions ${ }^{3}$ The preconcentration/ separation procedure is based on chelate formation of the metal ions like $\mathrm{Cu}(\mathrm{II}), \mathrm{Fe}(\mathrm{III}), \mathrm{Pb}(\mathrm{II}), \mathrm{Co}(\mathrm{II})$ and $\mathrm{Cr}(\mathrm{III})$ with benzopurpurine 4B and on retention of the chelates on cellulose nitrate membrane filter were studied for the determination of these metal ions by dissolving the membranes in acid. ${ }^{4}$ The heavy metal ions like copper, iron, cobalt, and cadmium were preconcentrated by collected on cellulose nitrate membrane filter as their 8-hydroxyquinoline complexes and the membrane filter was dissolved by using small amounts of concentrated nitric acid for their determinations. ${ }^{5}$ The main disadvantage of cellulose nitrate is its solubility in acidic conditions thus making it necessary to look for an alternate. Nafion with its high chemical stability appears as good alternate for metal ion uptake.

The structure of Nafion can be understood by different models.
Cluster-network model represents Nafion as consisting of sulphonate ion clusters in the form of inverted micelle with a diameter of $40 \AA$ within a continuous fluorocarbon lattice interconnected by narrow channels of $10 \AA$ in width. The other models namely core-shell model describes Nafion consisting of ion-rich core surrounded by an ion poor shell, a rod model where the sulfonic groups arrange into crystallike rods, and a sandwich model where the polymer forms two layers whose sulfonic groups attract across an aqueous layer where transport occurs. From all the models it is clear that there exists a network of ionic clusters. The degree of hydration is expected to affect the morphology of Nafion. Recent models show that the sulfonic acid functional groups self-organize into arrays of hydrophilic water channels of $2.5 \mathrm{~nm}$ diameter dispersed within hydrophobic polymer backbones and allow transport of small ions.

Ion exchange membranes find extensive applications due to their property of perm-selectivity which denotes the difference in permeability between the ions of opposite charges. It is to be mentioned that the perm-selectivity of a membrane is a function of various external parameters. Permeation of neutral substances, heterocyclic bases and cations..$^{6-8}$ Knowledge of the basic transport phenomena of ions in ion exchange membranes was important for the applications of these membranes. Pretreatment of Nafion membrane prior to its use in study has a strong effect on the ion exchange properties. ${ }^{9-11}$

In the present study, the uptakes of metal ions $\left(\mathrm{Cu}^{2+}\right.$ and $\left.\mathrm{Co}^{2+}\right)$ by Nafion under different experimental conditions have been studied. Here the role of permselectivity on the uptake property has been evaluated by studying in presence of various complexing agents. The studies were also carried out using Nafion pretreated differently to see the effect.

\section{Experimental}

Metal ion solutions were prepared by dissolving the appropriate weighed amounts of their chloride salts in dilute acid and standardized by complexometric titrations using suitable metallochromic indicators. The sodium acetate solution was prepared by dissolving $6.8 \mathrm{~g}$ of the salt (E. Merck GR) in $500 \mathrm{ml}$ of distilled water to give 
a stock solution of $0.1 \mathrm{M}$. This solution was used along with acetic acid for $\mathrm{pH}$ adjustments. EDTA and ethylenediamine solutions $(0.1 \mathrm{M})$ were prepared using appropriate amounts of the compounds obtained from BDH AnalaR.

Pretreatment of Nafion membranes were carried out by

(i) Immersing the Nafion pieces in $2 \mathrm{M} \mathrm{HCl}$ for $3 \mathrm{~h}$ at room temperature

(ii) Refluxing with $1: 1 \mathrm{HNO}_{3}$ for $1 \mathrm{~h}$, and then boiled with distilled water $\left(\sim 60^{\circ} \mathrm{C}\right)$ for $0.5 \mathrm{~h}$.

The membranes pretreated in the two different ways were labeled $\mathrm{N}$ and AT respectively. The experiments were carried out in batch mode. Weighed pieces (approximately $0.1 \mathrm{~g}$ ) of Nafion membrane, pre-treated differently were equilibrated with $10 \mathrm{~mL}$ of $0.01 \mathrm{M}$ of metal ion $\left(\mathrm{Cu}^{2+}\right.$ and $\left.\mathrm{Co}^{2+}\right)$ solution of known concentration for different periods. The amount taken up evaluated as a function of time.

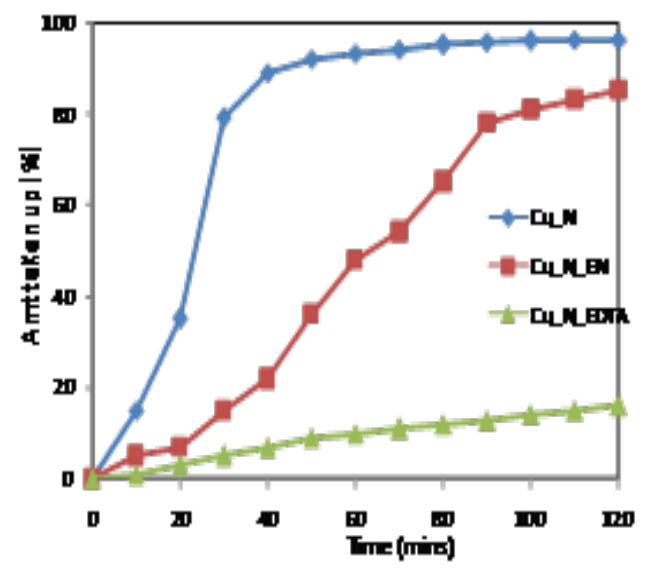

(A): N

\section{Results and discussion}

Nafion membrane has pendant $\mathrm{SO}_{3} \mathrm{H}$ groups is highly acidic and can be exchanged with other cations. Since Nafion is a cation exchange membrane, $\mathrm{pH}$ of the external solution would affect the uptake property of Nafion. From our earlier studies ${ }^{6-8}$ it was seen that for transition metal ions, the maximum uptake was in the $\mathrm{pH}$ range of 3.5-5.5 using sodium acetate buffer medium. Therefore in the present study, the $\mathrm{pH}$ was maintained in this range. Normally to achieve selectivity in separation, masking agents are used to complex the interfering ions. Thus it became of interest to see whether the organic complexing agents would affect the uptake property.

In the present study, the uptake of $\left(\mathrm{Cu}^{2+}\right.$ and $\left.\mathrm{Co}^{2+}\right)$ were carried out in presence of two complexing agents, ethylene diamine(EN) and ethylenediamine tetra acetic acid (EDTA). The results are shown in Figure $1 \& 2$. The batch mode experiments were carried out with Nafion membranes pretreated differently.

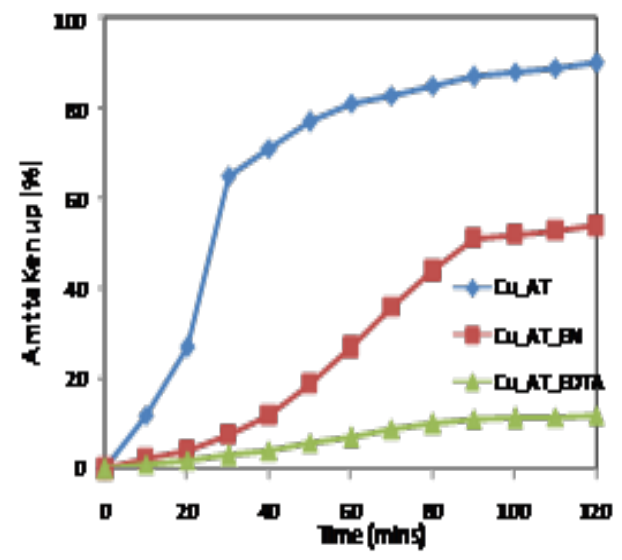

(B): $\mathbf{A T}$

Figure I Uptake of copper ions using differently pretreated membranes in presence of different organic complexing agents.

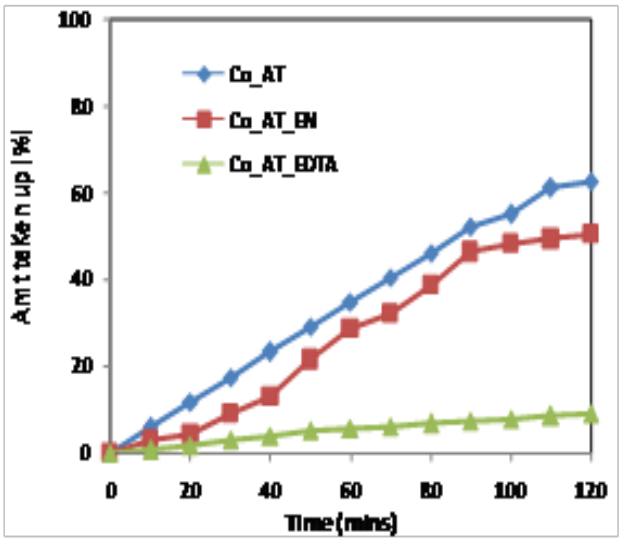

(A): $\mathrm{N}$

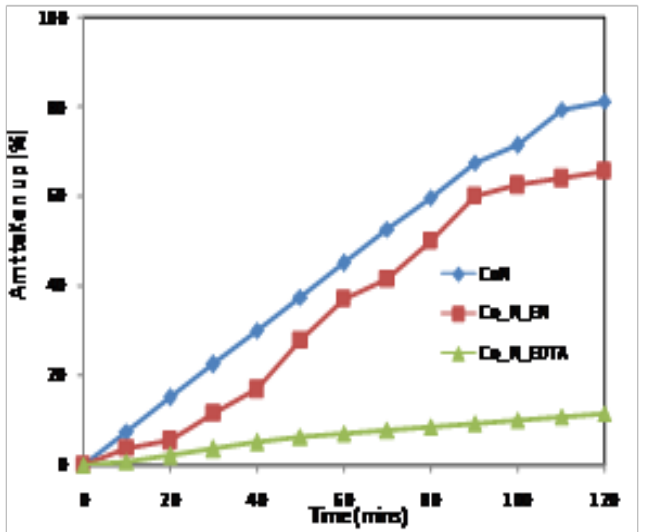

(B):AT

Figure 2 Uptake of cobalt ions using differently pretreated membranes in presence of different organic complexing agents.

From Figure 1, it is seen that the uptake of copper is affected by the pretreatment of membranes. It is seen that upon pretreatment with boiling acid, the water clusters become bigger and therefore results in lower uptake. This is in correlation with our earlier studies which indicated that the pretreatment resulted in the reduction of ion exchange capacity of the membrane..$^{6,7}$
The presence of organic complexing agents in the solution gives very interesting results. The uptake of copper from its pure solution is quite fast and nearly $80 \%$ uptake is completed within the first 30 mins. Then it reaches the maximum of $96 \%$. In presence of EN, the rate of uptake is quite low and the maximum amount taken up is $85 \%$. This is due to the fact that ethylenediamine will form a cationic 
complex with copper ions and therefore the amount of free copper ion in solution for uptake by Nafion is reduced. However with time, the amount taken up increases indicating a possibility of the copperethylenediamine complex being taken up. This is possible as the clusters would incorporate the copper ethylenediamine complex. The presence of EDTA in the solution decreases the uptake of copper ion. This is because copper forms an anionic complex and due to properties of permselectivity, Nafion does not take up the negatively charged complex. The small amount of copper in solution could probably be due to the competition of copper between the ion exchange group of

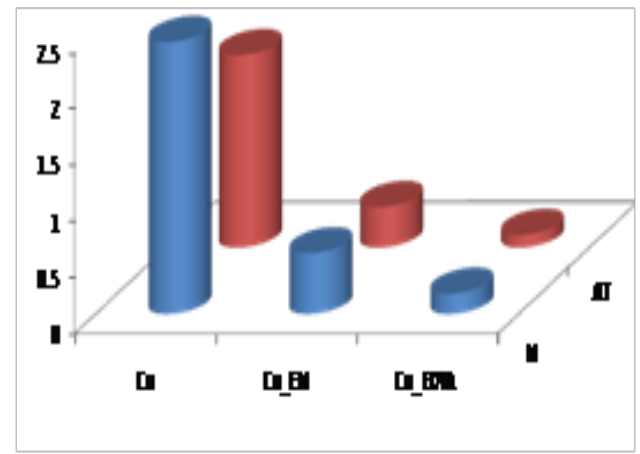

(A) Ca
Nafion and the EDTA present in solution.

The uptake of Cobalt using the membranes was carried out and the results are given in Figure 2. The uptake of cobalt shows a similar trend to that of copper but the overall amount taken up is lower. In order to understand the changes in the uptake of the metal ions, the rate of uptake was calculated and given in Figure 3. From Figure 3 it was seen that the rate constant values for copper uptake was higher than that of cobalt in all the different cases. This is due to the fact that Nafion has higher uptake capacity for copper as compared to cobalt.

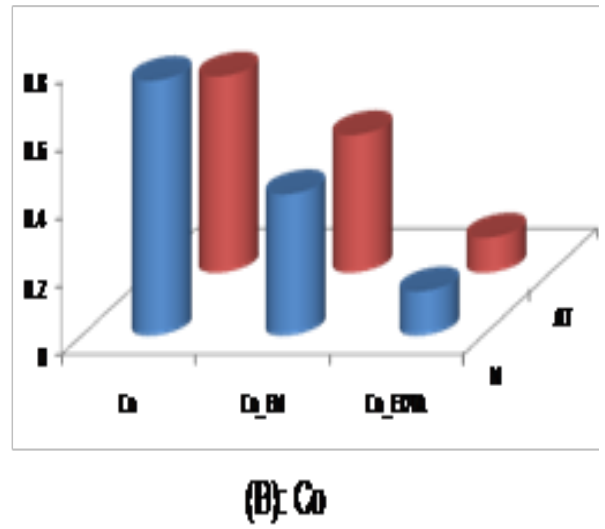

Figure 3 Rate constant variations for uptake of $\mathrm{Cu}$ and $\mathrm{Co}$ in presence of different organic complexing agents.

\section{Conclusion}

In the present study, effect of complexing agent (EN and EDTA) on the up take of transition metal ions ( $\mathrm{Cu}$ and $\mathrm{Co}$ ) was studied. The role of pretreatment of nafion membrane prior to its use was established. The formation of negatively charged complexes by EDTA results in a very low uptake of the metal ions. Ethylene diamine forms a cationic complex which is taken up by nafion. However the kinetics of this sorption is slower than that of pure metal ions. Pretreatment with boiling acids leads to the changes in the clusters present within the nafion resulting in difference in sorption properties.

\section{Acknowledgements}

None.

\section{Conflict of interest}

The author declares no conflict of interest.

\section{References}

1. Jayshree Ramkumar. 13-Nafion Perfluorosulphonate Membrane: Unique Properties and Various Applications. Functional Materials. 2012:549-577.

2. Ibrahim Narin, Mustafa Soylak. Enrichment and determinations of nickel (II), cadmium (II), copper (II), cobalt (II) and lead (II) ions in natural waters, table salts, tea and urine samples as pyrrolydine dithiocarbamate chelates by membrane filtration-flame atomic absorption spectrometry combination. Analytica Chimica Acta. 2003;493(2):205-212.

3. Soylak M, Divrikli U, Elci L, et al. Preconcentration of Cr(III), Co(II), $\mathrm{Cu}(\mathrm{II}), \mathrm{Fe}(\mathrm{III})$ and $\mathrm{Pb}(\mathrm{II})$ as calmagite chelates on cellulose nitrate membrane filter prior to their flame atomic absorption spectrometric determinations. Talanta. 2002;56(3):565-570.
4. Aslihan Uzun K, Mustafa S, Latif Elci. Separation/Preconcentration Of $\mathrm{Cu}(\mathrm{II}), \mathrm{Fe}(\mathrm{III}), \mathrm{Pb}(\mathrm{II}), \mathrm{Co}(\mathrm{II})$, And Cr(III) In Aqueous Samples On Cellulose Nitrate Membrane Filter And Their Determination By Atomic Absorption Spectrometry. Anal lett. 2002;35(9):1561-1574.

5. Soylak M, Narin I, Divrikli U, et al. Preconcentration-Separation of Heavy Metal Ions in Environmental Samples by Membrane Filtration-Atomic Absorption Spectrometry Combination. Anal lett. 2004;37(4):767780 .

6. Jayshree Ramkumar, Maiti B, Mathur PK. Use of Masking Agents in Promoting Selective Transport of $\mathrm{Zn}^{2+}$ through Nafion Membrane. Sep Sci Technol. 1998;33(15):2423-2429.

7. Jayshree Ramkumar, Unnikrishnan EK, Maiti B, et al. Facilitated transport of halides through Nafion ionomer membrane modified with lanthanide complexes. J Memb Sci. 1998;141(2):283-288.

8. Jayshree Ramkumar, Shrimal KS, Maiti B, et al. Selective permeation of $\mathrm{Cu}^{2+}$ and $\mathrm{UO}_{2}{ }^{2+}$ through a Nafion ionomer membrane. J Memb Sci. $1996 ; 116: 31-37$

9. JayshreeRamkumar, Tulsi Mukherjee. Role of Ion exchange in permeation processes. Talanta. 2007;71(3):1054-1060.

10. Jayshree Ramkumar, Tulsi M. Effect of aging on the water sorption and ion exchange studies on Nafion and Dowex resins: Transition metal ions-proton exchange systems. Sep Purif Technol. 2007;54(1):61-70.

11. Basu M, Jayshree Ramkumar. Understanding the Structural Changes in Nafion due to Shelf-Life and Pretreatment: Thermo Dynamical Approach of Water and Metal Ion Sorption. IJACS. 2014;3:19-24. 